\title{
CONSTANTS OF DE BRUIJN-NEWMAN TYPE IN ANALYTIC NUMBER THEORY AND STATISTICAL PHYSICS
}

\author{
CHARLES M. NEWMAN AND WEI WU
}

\begin{abstract}
One formulation in 1859 of the Riemann Hypothesis $(\mathrm{RH})$ was that the Fourier transform $H_{f}(z)$ of $f$ for $z \in \mathbb{C}$ has only real zeros when $f(t)$ is a specific function $\Phi(t)$. Pólya's 1920s approach to the RH extended $H_{f}$ to $H_{f, \lambda}$, the Fourier transform of $e^{\lambda t^{2}} f(t)$. We review developments of this approach to the $\mathrm{RH}$ and related ones in statistical physics where $f(t)$ is replaced by a measure $d \rho(t)$. Pólya's work together with 1950 and 1976 results of de Bruijn and Newman, respectively, imply the existence of a finite constant $\Lambda_{D N}=\Lambda_{D N}(\Phi)$ in $(-\infty, 1 / 2]$ such that $H_{\Phi, \lambda}$ has only real zeros if and only if $\lambda \geq \Lambda_{D N}$; the $\mathrm{RH}$ is then equivalent to $\Lambda_{D N} \leq 0$. Recent developments include the Rodgers and Tao proof of the 1976 conjecture that $\Lambda_{D N} \geq 0$ (that the $\mathrm{RH}$, if true, is only barely so) and the Polymath 15 project improving the $1 / 2$ upper bound to about 0.22 . We also present examples of $\rho$ 's with differing $H_{\rho, \lambda}$ and $\Lambda_{D N}(\rho)$ behaviors; some of these are new and based on a recent weak convergence theorem of the authors.
\end{abstract}

\section{INTRODUCTION}

For $\rho$ a positive Borel measure on the real line with $\rho(-\infty,+\infty)<\infty$ and $\lambda$ real, define

$$
H_{\rho, \lambda}(z):=\int_{-\infty}^{\infty} e^{i z t} e^{\lambda t^{2}} d \rho(t)
$$

When $\rho$ has a density $f(t)$ with respect to Lebesgue measure, this becomes

$$
H_{f, \lambda}(z):=\int_{-\infty}^{\infty} e^{i z t} e^{\lambda t^{2}} f(t) d t .
$$

Depending on the behavior of $d \rho$ (or $f$ ) as $t \rightarrow \pm \infty, H_{\rho, \lambda}(z)$ will be an entire function of complex $z$ either for all real $\lambda$ or for a semi-infinite interval of $\lambda$ including at least $(-\infty, 0)$. The focus of this paper is on the subset $\mathcal{P}_{\rho}$ (or $\left.\mathcal{P}_{f}\right)$ of those $\lambda$ such that $H_{\rho, \lambda}$ (or $H_{f, \lambda}$ ) is entire and all its zeros in the complex plane are purely real.

The main interest in this question, from analytic number theory, is its relation to the Riemann Hypothesis (RH). Taking $f$ to be a specific function $\Phi$ (see (7) below), $\mathrm{RH}$ is equivalent to all the zeros of $H_{\Phi, 0}$ being real-i.e., equivalent to $\mathcal{P}_{\Phi} \ni 0$. In section 2 we review, following the history from 1859 Rie59] to the present RoT18, some of the main developments of this approach to $\mathrm{RH}$, including (see subsections

Received by the editors March 3, 2019.

2010 Mathematics Subject Classification. Primary 11M26, 30C15, 60K35.

The research reported here was supported in part by US NSF grant DMS-1507019. 
2.52 2.6) those with motivations arising from statistical physics. Three key facts are the following.

(1) $\lambda \in \mathcal{P}_{\Phi}$ and $\lambda^{\prime}>\lambda$ imply $\lambda^{\prime} \in \mathcal{P}_{\Phi}$; this is due to Pólya P27 - see Theorem 2 .

(2) $1 / 2 \in \mathcal{P}_{\Phi}$; this is due to De Bruijn DB50 - see Theorem 7

(3) $\mathcal{P}_{\Phi}$ is bounded below; this is due to Newman [Ne76] see Theorem 9 ,

It follows from these three facts that there is a well-defined constant in $(-\infty, 1 / 2]$, now known as the de Bruijn-Newman constant, which (for the case $f=\Phi$ ) we denote simply as $\Lambda_{D N}$, so that $\mathcal{P}_{\Phi}=\left[\Lambda_{D N}, \infty\right)$ and $R H$ is equivalent to $\Lambda_{D N} \leq 0$. In Ne76, the proof that $\Lambda_{D N}>-\infty$ (i.e., see (3) above) was accompanied by the conjecture, complementary to $\mathrm{RH}$, that $\Lambda_{D N} \geq 0$, stated there as a "quantitative dictum that the Riemann Hypothesis, if true, is only barely so".

In subsection 2.6 we survey the history, starting with CNV88, of improvements on the [Ne76] lower bound $\Lambda_{D N}>-\infty$ culminating with the 2018 proof by Rodgers and Tao RoT18, of the 1976 conjecture that $\Lambda_{D N} \geq 0$. Then in subsection 2.7 we discuss the shorter history of improvements to the [DB50] upper bound $\Lambda_{D N} \leq 1 / 2$, consisting of the Ki-Kim-Lee KKL09] bound $\Lambda_{D N}<1 / 2$ (and related results such as Theorem 14 below) as well as current efforts (see [T18]) by Tao and collaborators to obtain a concrete upper bound in $(0,1 / 2)$, expected at the time of this writing to be about 0.22 .

In section 3 of this paper we go beyond the RH case of $d \rho=\Phi(x) d x$ and discuss a number of results and examples about $\mathcal{P}_{\rho}$ or $\mathcal{P}_{f}$ for more general $\rho$ and $f$. This is natural from the statistical or mathematical physics point of view and may be of general mathematical interest even without an immediate connection to analytic number theory. As in the case of $\Phi$, we will always assume that $\rho$ and $f$ are nonnegative and even; sometimes $\rho$ will be normalized to be a probability measure.

A key result (see Theorem 16) discussed in subsection 3.1 is a somewhat surprising weak convergence theorem from [NeW17] for sequences of probability distributions whose Fourier transforms have only purely real zeros. The surprising aspect of this theorem is that the purely real zeros property somehow controls tail behavior of the sequence of distributions in a uniform way. We make use of this theorem several times in section [3, including in subsection 3.2 where we classify and give examples of measures $\rho$ with varying tail behaviors and varying $\mathcal{P}_{\rho}$ 's. The tail behaviors are subdivided in the three subsections of 3.2 according to whether

$$
\mathcal{T}_{\rho}:=\left\{b \in(-\infty,+\infty): \int e^{b x^{2}} d \rho<\infty\right\}
$$

is $(-\infty, \infty)$ or $\left(-\infty, b_{0}\right)$ or $\left(-\infty, b_{0}\right]$.

All possibilities for the pair $\left(\mathcal{T}_{\rho}, \mathcal{P}_{\rho}\right)$ are presented in subsection 3.2 in nine classes, labeled Cases 1 through 9. Concrete examples of a $\rho$ for each class are provided, with the notable exception of Case 7 where it is shown that one cannot have $\mathcal{T}_{\rho}=\left(-\infty, b_{0}\right]$ and $\mathcal{P}_{\rho}$ strictly bigger than $\left\{b_{0}\right\}$; this is an interesting consequence of the weak convergence result, Theorem 16. Another consequence of Theorem 16 is the construction in Case 3 of a $\rho$ with $\mathcal{T}_{\rho}=(-\infty, \infty)$ but with $\mathcal{P}_{\rho}=\emptyset$ - see, in particular, Proposition 19.

We conclude this introduction by noting that there are other connections between the Riemann zeta function and statistical physics than those we discuss. For example, random matrix theory seems to describe very accurately the distribution of 
the zeros (as stated by Montgomery's conjecture [Mon73]) and the fine asymptotics of the moments; see for example [BK13] for a review. More recently, Fyodorov and Keating [FK14] also suggested that the extreme values of the zeta function in short intervals behave as a log-correlated Gaussian field. See [ABB19, Naj18, AOR19] for recent progress on these questions.

\section{History of the de Bruijn-Newman constant}

2.1. The Riemann Hypothesis and zeros of Fourier transforms. In his fundamental paper Rie59, Riemann extended into the complex plane the $\zeta$ function, defined originally by Euler as

$$
\zeta(s)=\sum_{n \geq 1} n^{-s}
$$

for real $s>1$. Obviously, $\zeta$ is holomorphic in the domain $\{\Re s>1\}$. The $\zeta$ function is closely related to the distribution of primes, through Euler's product expansion, $\sum_{n \geq 1} n^{-s}=\prod_{p}\left(1-p^{-s}\right)^{-1}$, over primes $p \geq 2$. A main achievement of [Rie59] is that the function $\zeta$ admits an analytic continuation to the whole complex plane (except for a simple pole at $s=1$ ), and the location of the zeros of $\zeta$ are closely related to the asymptotic behavior as $x \rightarrow \infty$ of $\pi(x)$, the number of primes less than or equal to $x$.

We now sketch some of the basic ideas of [Rie59]. Starting with the identity for the Gamma function $\Gamma(z)$,

$$
\int_{0}^{\infty} \exp \left(-\pi n^{2} x\right) x^{\frac{s}{2}-1} d x=\frac{\Gamma(s / 2)}{\pi^{\frac{s}{2}} n^{s}}, \quad \Re s>0, n \in \mathbb{N},
$$

one obtains

where

$$
\pi^{-\frac{s}{2}} \Gamma\left(\frac{s}{2}\right) \zeta(s)=\int_{0}^{\infty} \psi(x) x^{\frac{s}{2}-1} d x
$$

$$
\psi(x)=\sum_{n=1}^{\infty} \exp \left(-\pi n^{2} x\right) .
$$

Notice that $\theta(x):=2 \psi(x)+1$ is the well-known Jacobi-theta function, which satisfies the identity

$$
\theta(x)=x^{-\frac{1}{2}} \theta\left(x^{-1}\right) .
$$

The identity follows from the Poisson summation formula, which gives

$$
\begin{aligned}
\theta(x)=\sum_{n=-\infty}^{\infty} \exp \left(-\pi n^{2} x\right)=\sum_{k=-\infty}^{\infty} \int_{-\infty}^{\infty} e^{-\pi t^{2} x} e^{-2 \pi i k t} d t \\
\quad=\sum_{k=-\infty}^{\infty} \frac{e^{-\pi k^{2} / x}}{\sqrt{x}}=x^{-\frac{1}{2}} \theta\left(x^{-1}\right) .
\end{aligned}
$$

Riemann used (4) to obtain the representation

$$
\pi^{-\frac{s}{2}} \Gamma\left(\frac{s}{2}\right) \zeta(s)=\frac{1}{s(s-1)}+\int_{1}^{\infty} \psi(x)\left(x^{\frac{s}{2}-1}+x^{\frac{1-s}{2}-1}\right) d x, \quad \Re s>1 .
$$

Note that the integral above is uniformly convergent on compact subsets of $\mathbb{C}$, and therefore the left-hand side admits an analytic continuation in $\mathbb{C} \backslash\{0,1\}$. The function $\Gamma\left(\frac{s}{2}\right)$ has a pole at 0 , and simple poles at $-2,-4, \ldots$; thus the values $-2 k$, 
$k \in \mathbb{N}$, are simple zeros of $\zeta$, known as the trivial zeros. Moreover, $\zeta$ does not vanish at other points in the half-planes $\Re s<0$ or $\Re s>1$. All other possible zeros of $\zeta$ are in the strip $0 \leq \Re s \leq 1$, called the critical strip.

Using the change of variable $s=\frac{1}{2}+i z$, Riemann also introduced the $\xi$ function as

$$
\xi(z)=s(s-1) \Gamma\left(\frac{s}{2}\right) \pi^{-\frac{s}{2}} \zeta(s) .
$$

It follows from (44) and the above discussion that $\xi$ is an even entire function. Furthermore, all possible zeros of $\xi$ lie in the strip $|\Im z| \leq \frac{1}{2}$.

In Rie59] Riemann conjectured that $\zeta$ has infinitely many zeros in the critical strip, and that one can explicitly represent

$$
\pi(x)=l i(x)+\sum_{\rho \in \mathcal{Z}, \Im \rho>0}\left(l i\left(x^{\rho}\right)+l i\left(x^{1-\rho}\right)\right)+\int_{x}^{\infty} \frac{d t}{\left(t^{2}-1\right) \log t}-\log 2, x \geq 2,
$$

where $\operatorname{li}(x):=\int_{1}^{x}(\ln t)^{-1} d t$ (with the integral defined at $t=1$ in the principal value sense) and $\mathcal{Z}$ is the set of zeros of $\zeta$ in the critical strip. This formula was later rigorously established by von Mangoldt. Riemann further conjectured that the zeros of the $\xi$ function are all purely imaginary (or equivalently, all nontrivial zeros of $\zeta$ are on the line $\Re s=\frac{1}{2}$ ). This is the celebrated Riemann Hypothesis.

Using (5), (6), and a change of variables, we have

$$
\xi(z)=2 \int_{0}^{\infty} \Phi(u) \cos z u d u
$$

where

$$
\Phi(u)=\sum_{n \geq 1}\left(4 \pi^{2} n^{4} e^{9 u / 2}-6 \pi n^{2} e^{5 u / 2}\right) e^{-\pi n^{2} \exp (2 u)} .
$$

It is not obvious that $\Phi(u)$ is even, but in fact it is, as follows from (4). Then the $\xi$ function is just the Fourier transform of $\Phi$ :

$$
\xi(z)=\int_{-\infty}^{\infty} e^{i z u} \Phi(u) d u
$$

It is not hard to see that $\Phi(u) \geq 0$ for all $u$; this positivity is valid term by term on the right-hand side of (7) for $u \geq 0$, and then the evenness of $\Phi$ implies positivity for $u \leq 0$. Up to a multiplicative constant, $\Phi$ may be regarded as an even probability density function.

Based on the considerations above, a natural approach to the Riemann Hypothesis is to establish criteria for Fourier transforms of (sufficiently rapidly decreasing at $\pm \infty$ ) probability densities to possess only real zeros, and to apply them to the Riemann $\xi$ function. As will be discussed later, this encouraged study of the distribution of zeros of Fourier transforms.

2.2. Pólya and universal factors. Motivated by the Riemann Hypothesis, Pólya systematically investigated the question of when the Fourier transform of a function has only real roots (or equivalently, the Laplace transform has only pure imaginary zeros). The function $\Phi$ related to the Riemann $\xi$ function seems complicated, so Pólya's starting point was to use (relatively simple) classes of entire functions whose zeros are all real.

In [PSc14, by applying a theorem of Laguerre, the authors Pólya and Schur obtained the following class of entire functions, whose zeros are all real: 
Theorem 1. The entire function $f(z)$ is a uniform limit of real polynomials with only real zeros, if and only if

$$
f(z)=b z^{n} \exp \left(-\lambda z^{2}+\kappa z\right) \prod_{k}\left(1-\frac{z}{b_{k}}\right) \exp \left(\frac{z}{b_{k}}\right),
$$

where $b, \kappa \in \mathbb{R}, n \in \mathbb{N}, \lambda \geq 0, b_{k} \in \mathbb{R}$, the product can be finite or infinite and $\sum_{k} b_{k}^{-2}<\infty$.

Functions of the form (8) are said to belong to the Laguerre-Pólya class and we write $f \in \mathcal{L} \mathcal{P}$. The Riemann Hypothesis is equivalent to the statement that $\xi \in \mathcal{L} \mathcal{P}$. At the time, there was a hope that a complete characterization of the functions in $\mathcal{L P}$ could lead to a proof (or disproof) of the Riemann Hypothesis. However, the conditions for functions to belong to $\mathcal{L P}$ were too involved to be applied to the Riemann $\xi$ function, and this approach was slowly abandoned.

Later in [P27, Pólya investigated when entire functions of the form

$$
f(z):=\int_{-\infty}^{\infty} F(t) e^{i z t} d t
$$

have only real zeros. The complex-valued function $F$ is assumed to be locally integrable and to satisfy the conditions

$$
F(-t)=\bar{F}(t) \quad \forall t
$$

and, for all $t$,

$$
|F(t)| \leq A \exp \left(-|t|^{2+\alpha}\right), \quad \text { for some } A, \alpha>0 .
$$

To prove his results, Pólya introduced the notion of universal factors. These are complex-valued functions $\varphi(t)$ for $t \in \mathbb{R}$, such that if (9) has only real zeros, then the Fourier transform

$$
\int_{-\infty}^{\infty} \varphi(t) F(t) e^{i z t} d t
$$

also has only real zeros. Pólya obtained the following complete characterization of universal factors.

Theorem 2. A real analytic function $\varphi(t), t \in \mathbb{R}$, is a universal factor if and only if its holomorphic extension $\varphi(t)$ in $\mathbb{C}$ is such that $\varphi(i z)$ is an entire function of the form (8).

Taking $f(z)=e^{-\lambda z^{2}}$ in (8), a simple example of a universal factor is $\varphi(t)=e^{B t^{2}}$ with $B>0$. In other words, if all roots of

$$
\int_{-\infty}^{\infty} e^{i z t} F(t) d t
$$

are real, then the same holds for $\int_{-\infty}^{\infty} e^{i z t} e^{B t^{2}} F(t) d t$ for $B>0$. Another simple example of a universal factor is $\varphi(t)=\cosh a t$.

Using Theorem 2 Pólya obtained the following class of functions that have only real zeros. 
Theorem 3. Suppose that $F$ satisfies (10) and (11), and in addition, has a holomorphic extension in a neighborhood of the origin. Then the complex function

$$
H(z)=\int_{0}^{\infty} F(t) t^{z-1} d t
$$

admits an analytic continuation as a meromorphic function in $\mathbb{C}$. If $H$ does not have zeros in the region $\mathbb{C} \backslash(-\infty, 0]$ and $q \in \mathbb{N}$, then the entire function

$$
\int_{-\infty}^{\infty} F\left(t^{2 q}\right) e^{i z t} d t
$$

has only real zeros.

By applying Theorems 2 and 3 Pólya concluded that the entire functions

$$
\begin{aligned}
& \int_{-\infty}^{\infty} \exp (-a \cosh t) e^{i z t} d t \\
& \int_{-\infty}^{\infty} \exp \left(-t^{2 q}\right) e^{i z t} d t
\end{aligned}
$$

have only real zeros and so does the entire function

$$
\int_{-\infty}^{\infty} \exp \left(-a t^{4 q}+b t^{2 q}+c t^{2}\right) e^{i z t} d t, \quad q \in \mathbb{N} .
$$

In another paper of Pólya [P26], he explained how the existence of infinitely many real zeros is in general easier to prove than the nonexistence of complex zeros. In particular, a method of Hardy leads to the following criterion:

Proposition 4. Suppose that $F$ is an even function, analytic and real, and such that $\lim _{t \rightarrow \infty} F^{(n)}(t) t^{2}=0$ for $n=0,1,2, \ldots$ If the function $f(z)$ defined by (9) has only a finite number of real zeros, then there is an integer $N$ such that $\left|F^{(n)}(i t)\right|$ is an increasing function if $n>N$ and $0<t<T$, where iT is the singular point of $F(t)$ which is closest to the origin.

2.3. De Bruijn's results. In DB50, de Bruijn continued Pólya's line of research and studied the zeros of entire functions of the form (9), under the conditions (10) and (11). Examples of such entire functions include

$$
\int_{-\infty}^{\infty} e^{-t^{2 n}} e^{i z t} d t, \quad n \in \mathbb{N}
$$

and

$$
\int_{-\infty}^{\infty} \exp (-a \cosh t) e^{i z t} d t, \quad a>0 .
$$

Two of the main results of [DB50] are the next two theorems.

Theorem 5. Let $f(t)$ be an entire function such that its derivative $f^{\prime}(t)$ is the limit (uniform in any bounded domain) of a sequence of polynomials, all of whose roots lie on the imaginary axis. Suppose further that $f$ is not a constant, $f(t)=f(-t)$, and $f(t) \geq 0$ for $t \in \mathbb{R}$. Then the integral

$$
\int_{-\infty}^{\infty} \exp (-f(t)) e^{i z t} d t
$$

has real roots only.

The proof of this result relies on the following. 
Theorem 6. Let $N \in \mathbb{N}$, and let

$$
P(t)=\sum_{n=-N}^{N} p_{n} e^{n t}, \quad \Re p_{n}>0, p_{-n}=\bar{p}_{n}, n=1, \ldots, N .
$$

Let the function $q(z)$ be regular in the sector $-\pi / 2 N-N^{-1} \arg p_{N}<\arg z<$ $\pi / 2 N-N^{-1} \arg p_{N}$ and on its boundary, with the possible exception of $z=0$ and $z=\infty$, which may be poles (of arbitrary finite order) for $q(z)$. Furthermore, suppose $\bar{q}(z)=q(1 / \bar{z})$ in this sector (in other words, $q(z)$ is real for $|z|=1$ ). Then all but a finite number of roots of the function

$$
\Psi(z)=\int_{-\infty}^{\infty} \exp (-P(t)) q\left(e^{t}\right) e^{i z t} d t
$$

are real.

A key ingredient used to prove Theorems 5] and [6] was the notion of a strong universal factor. Suppose that $F$ satisfies (10) and (11), and the complex function $S(t)$ satisfies the following two properties.

(1) If the roots of (9) lie in a strip $|\Im z| \leq \Delta$, for some $\Delta>0$, then those of

$$
\int_{-\infty}^{\infty} F(t) S(t) e^{i z t} d t
$$

lie in a strip $|\Im z| \leq \Delta_{1}$, where the constant $\Delta_{1}<\Delta$ is independent of $F$.

(2) If, for any $\varepsilon>0$, all but a finite number of roots of (9) lie in the strip $|\Im z| \leq \varepsilon$, then the function (15) has only a finite number of nonreal roots.

Then $S(t)$ is called a strong universal factor. By definition, any strong universal factor is a universal factor in Pólya's sense. The function $S(t)=\cosh t$ is a simple example of a strong universal factor. As discussed earlier, the Gaussian density $e^{B t^{2}}$ is a universal factor, and the following theorem of DB50 shows it has Property 1. It was later proved in [KKL09] to also have Property 2.

Theorem 7. Suppose that the function F satisfies (10) and (11), and the zeros of the entire function (9) lie in the strip $|\Im z| \leq \Delta$. Then all the roots of the entire function

$$
\int_{-\infty}^{\infty} F(t) e^{\lambda t^{2} / 2} e^{i z t} d t
$$

lie in the strip

$$
|\Im z| \leq\left[\max \left(\Delta^{2}-\lambda, 0\right)\right]^{1 / 2} .
$$

De Bruijn also showed that a large class of functions are strong universal factors. In particular, functions of the type

$$
S(t)=\sum_{n=-N}^{N} a_{n} e^{n \lambda t}, \quad a_{n}=\bar{a}_{-n}, \lambda>0,
$$

are strong universal factors if all their roots lie on the imaginary axis. Conversely, if a function of the form (18) is a strong universal factor, then all of its roots lie on the imaginary axis. In particular, de Bruijn proved the following. 
Theorem 8. Suppose the roots of the function (18) lie on the imaginary axis. Then if the roots (resp., all but a finite number of the roots) of (9) lie in the strip $|\Im z| \leq \Delta$, then the roots (resp., all but a finite number of the roots) of (15) lie in the strip $|\Im z| \leq\left[\max \left(\Delta^{2}-\lambda^{2} N / 2,0\right)\right]^{1 / 2}$.

2.4. The de Bruijn-Newman constant. Here is a direct consequence of Theorem 7. Recall that the function $\Phi$ in (7) is (proportional to) a probability density related to the Riemann $\xi$ function. Define for any $\lambda \in \mathbb{R}, z \in \mathbb{C}$,

$$
H_{\lambda}(z)=\int_{-\infty}^{\infty} e^{\lambda t^{2}} \Phi(t) e^{i z t} d t .
$$

Applying Pólya's results (see the discussion following Theorem 2 above), it follows that there exists a constant $\Lambda_{D N}$ (but at this stage of our discussion, it could potentially be $\pm \infty$ ), such that $H_{\lambda}$ has all real zeros if and only if $\lambda \geq \Lambda_{D N}$. Moreover, since the roots of $\xi$ lie in the strip $|\Im z| \leq \frac{1}{2}$, de Bruijn's quantitative bound (17) gives an upper bound $\Lambda_{D N} \leq 1 / 2$.

More generally, one can define the functions $H_{f, \lambda}$ for a density $f$ and $H_{\rho, \lambda}$ for a measure $\rho$ by (2) and (11) and then a corresponding $\Lambda_{D N}(f)$ or $\Lambda_{D N}(\rho)$. This will be the topic of section 3 below.

We return to the constant $\Lambda_{D N}$ defined after equation (19). The result of de Bruijn that $\Lambda_{D N} \leq 1 / 2$ did not exclude the possibility that $\Lambda_{D N}=-\infty$. The first lower bound for $\Lambda_{D N}$ was given in [Ne76]:

Theorem 9. There exists a real number $\Lambda_{D N}$ with $-\infty<\Lambda_{D N} \leq 1 / 2$, such that $H_{\lambda}$ defined by (19) has only real zeros when $\lambda \geq \Lambda_{D N}$ but has nonreal zeros when $\lambda<\Lambda_{D N}$.

The constant $\Lambda_{D N}$ is now known as the de Bruijn-Newman constant. By definition, the Riemann Hypothesis is equivalent to having the zeros of $H_{0}$ all purely real. Therefore it is equivalent to $\Lambda_{D N} \leq 0$. In [Ne76], the complementary conjecture was made that $\Lambda_{D N} \geq 0$, as a quantitative version of the notion that "the Riemann Hypothesis, if true, is only barely so".

Theorem 9 was proved in Ne76] by giving a complete characterization of all even probability measures $\rho$ such that for any $\lambda>0$ the Fourier transform of $\exp \left(-\lambda t^{2}\right) d \rho(t)$ has only real zeros, as stated in the next theorem. It may be of some interest to note that the original motivation for this result was provided more by statistical physics and Euclidean field theory (as discussed in section 2.5) than by analytic number theory.

Theorem 10. Let $\rho$ be an even probability measure, and let $H_{\rho, \lambda}$ be defined by (1). Then $H_{\rho, \lambda}$ has only real zeros for all $\lambda \in \mathbb{R}$ if and only if either

$$
d \rho(t)=\frac{1}{2}\left(\delta\left(t-t_{0}\right)+\delta\left(t+t_{0}\right)\right), \text { for some } t_{0} \geq 0,
$$

or $d \rho=f(t) d t$ with

$$
f(t)=K t^{2 m} \exp \left(-\alpha t^{4}-\beta t^{2}\right) \prod_{j}\left[\left(1+\frac{t^{2}}{a_{j}^{2}}\right) e^{-\frac{t^{2}}{a_{j}^{2}}}\right] .
$$

Here $K>0, m=0,1, \ldots, a_{j}>0, \sum \frac{1}{a_{j}^{4}}<\infty, \alpha>0$, and $\beta \in \mathbb{R}($ or $\alpha=0$ and $\left.\beta+\sum \frac{1}{a_{j}^{2}}>0\right)$. 
Theorem 9 follows from Theorem 10 by showing that $\Phi(t)$ is not of the form (20). This was done in Ne76 by using the elementary inequality

$$
\left(1+x^{2}\right) e^{-x^{2}} \geq e^{-x^{4} / 2}
$$

for real $x$, to show that $f(t)$ given by (20) satisfies

$$
f(t) \geq K t^{2 m} \exp \left(-\left[\alpha+\sum_{j} 1 /\left(2 a_{j}^{4}\right)\right] t^{4}-\beta t^{2}\right),
$$

which decays much more slowly as $|t| \rightarrow \infty$ than does $\Phi(t)$.

When $\alpha=0$ in the above expression for $f(t), H_{f, \lambda}$ is required to have only real zeros for those $\lambda$ such that $H_{f, \lambda}$ is an entire function. For example, if there is only a finite product over $j$ in (20), then those are the $\lambda$ in $\left(-\infty, \beta+\sum\left(1 / \alpha_{j}^{2}\right)\right)$.

It is worth noting that the density (20) is reminiscent of the Laguerre-Pólya class (8). Also, (20) has a natural physical interpretation - the theorem implies that in order to have $\Lambda_{D N}=-\infty, f$ can only be either a discrete density with two point masses, or a density that is a kind of perturbation of the $\phi^{4}$ measure (i.e., the $q=1$ case of (13) $)$. See the next section for more details.

\subsection{Zeros of the partition function in statistical mechanics and quan-} tum field theory. In this subsection we take a detour and discuss examples of probability distributions arising in statistical mechanics and Euclidean (quantum) field theory, whose Laplace transform has only pure imaginary zeros. In the 1950s, Yang and Lee studied partition functions of a lattice gas with a variable chemical potential, or equivalently, of an Ising model in a variable external magnetic field [YL52,LY52]. They discovered a striking property for the locations of zeros of the Ising partition function - all the roots in the complex plane for the magnetic field variable lie on the imaginary axis. To be more precise, consider a collection of \pm 1 -valued random variables $\left\{X_{j}\right\}_{j=1}^{N}$ whose joint distribution is given by the Gibbs measure

$$
d \mu=K \exp \left(\sum_{i, j=1}^{N} J_{i j} x_{i} x_{j}\right) \prod_{j=1}^{N}\left(\delta\left(x_{j}-1\right)+\delta\left(x_{j}+1\right)\right)
$$

where $J_{i j} \geq 0$ and $K$ is the normalizing constant that makes (21) a probability measure. Then

$$
\int \exp \left(i t \sum_{i=1}^{N} \lambda_{i} x_{i}\right) d \mu, \quad \lambda_{i} \geq 0,
$$

as a function of complex $t$ has only real zeros. The original proof, in LY52, expressed this transform as a multinomial in the variables $\left\{e^{ \pm i t \lambda_{j}}\right\}$ and used fractional linear transformation arguments. Quite different proofs may be found; see [Ne74, Sec. 3] and [LS81].

The result of Lee and Yang has been extended to more general Ising-type models in which $\delta\left(x_{j}-1\right)+\delta\left(x_{j}+1\right)$ is replaced by some other measure $d \mu_{0}(x)$. For example, in the study of lattice $\phi^{4}$ Euclidean field theories, $d \mu_{0}(\phi)$ would be of the form $\exp \left(-a \phi^{4}+b \phi^{2}\right) d \phi$; a good general reference for this topic is FFS92. One of the extensions of the Lee-Yang result, established in [Ne74] (see also [LS81]), is as follows. 
Theorem 11. Suppose the Hamiltonian, $H(S)$ for $S \in \mathbb{R}^{N}$ is

$$
H(S)=-\sum_{i, j=1}^{N} J_{i j} S_{i} S_{j}
$$

with $J_{i j}=J_{j i} \geq 0, \forall i, j$, and $\mu_{0}$ is an arbitrary signed measure on $\mathbb{R}$ that is even or odd, has the property that $\int_{-\infty}^{\infty} e^{b s^{2}} d\left|\mu_{0}(s)\right|<\infty$ for all $b$, and satisfies the condition

$$
\int_{-\infty}^{\infty} e^{h s} d \mu_{0}(s) \neq 0, \quad \text { if } \Re h>0 .
$$

Then, for all $\beta \geq 0$,

$$
\int e^{\beta \sum h_{i} s_{i}} e^{-\beta H(S)} \prod_{i=1}^{N} d \mu_{0}\left(s_{i}\right) \neq 0, \quad \text { if } \Re h_{i}>0 \forall i .
$$

It follows that

$$
F(z):=\int e^{i z \sum \lambda_{i} s_{i}} e^{-\beta H(S)} \prod_{i=1}^{N} d \mu_{0}\left(s_{i}\right), \text { with } \lambda_{i} \geq 0 \forall i,
$$

has only real zeros. If the integral property above is only valid for small $b>0$, then the conclusions remain valid, but only for small $\beta>0$.

Lee-Yang type theorems also arise in the context of constructive quantum field theory. Indeed, an important special case of Theorem 11 is when

$$
d \mu_{0}(s)=\exp \left(-a s^{4}-b s^{2}\right) d s, a>0 \text { and } b \in \mathbb{R} .
$$

Theorem 11] when combined with Pólya's result about (13) with $q=1$ then implies the Lee-Yang property for continuum $\left(\phi^{4}\right)$ fields. This was actually first established by Simon and Griffiths SiG73] in a different way using an approximation by classical Ising models. In the same paper, they also showed the Lee-Yang property fails for some measures of the form

$$
d \mu(s)=\exp \left(-a s^{6}-b s^{4}-c s^{2}\right) d s, \quad a>0 .
$$

Some related results concerning a certain correlation inequality for Ising models and quantum field theories and its relation to the Riemann hypothesis may be found in $\mathrm{Ne91}$.

2.6. Lower bounds for $\Lambda_{D N}$ and the proof of the Ne76 conjecture that $\Lambda_{D N} \geq 0$. Improvements to the [Ne76] result that $\Lambda_{D N}>-\infty$ have been made since the late 1980s, giving a series of quantitative lower bounds for $\Lambda_{D N}$ that supported the conjecture that $\Lambda_{D N} \geq 0$. The first result in this direction was by Csordas, Norfolk, and Varga CNV88, who proved $\Lambda_{D N} \geq-50$. This was followed by R91, NRV92, CRV91 and then a paper by Csordas, Smith, and Varga CSV94, which showed $\Lambda_{D N} \geq-4.379 \times 10^{-6}$. There were further improvements in COSV93, O00, and then by Saouter, Gourdon, and Demichel [SGD11 who showed $\Lambda_{D N} \geq-1.15 \times 10^{-11}$.

We briefly summarize the methodology behind these proofs, in particular the approach of CSV94. Assume for now $\Lambda_{D N}<0$ and so that also the Riemann Hypothesis holds. The lower bound for $\Lambda_{D N}$ in CSV94 was established by exploiting the following repulsion phenomenon: if $\Lambda_{D N}$ were significantly less than zero, then adjacent zeros of $H_{0}$ (or $\xi$ ) could not be too close to each other, which 
would contradict known facts about close pairs of zeros of $\xi$. More precisely, let $0<x_{1}<x_{2}<\cdots$ be the nonnegative zeros of $\xi$, and let $x_{-j}=-x_{j}$. Define

$$
g_{k}:=\sum_{j \neq k, k+1}\left[\frac{1}{\left(x_{k}-x_{j}\right)^{2}}+\frac{1}{\left(x_{k+1}-x_{j}\right)^{2}}\right] \text {. }
$$

The following lower bound is established in CSV94.

Theorem 12. The de Bruijn-Newman constant satisfies

$$
\forall k, \Lambda_{D N} \geq \lambda_{k}
$$

where

$$
\lambda_{k}:=\frac{\left(1-\frac{5}{4}\left(x_{k+1}-x_{k}\right)^{2} g_{k}\right)^{4 / 5}-1}{8 g_{k}} .
$$

Then lower bounds for $\Lambda_{D N}$ can be obtained by studying Lehmer pairs of zeros Leh56, which are, roughly speaking, two consecutive simple zeros on the critical line that are exceptionally close to each other. The results of CSV94] and SGD11] were obtained by first numerically locating Lehmer pairs of increasingly high quality. In principle, by locating infinitely many Lehmer pairs with arbitrary close gaps, the method of CSV94 could verify the conjecture $\Lambda_{D N} \geq 0$. However, known upper bounds on gaps of zeros of $\xi$ were not sufficient to make that strategy work.

More recently, Rodgers and Tao RoT18 proved that $\Lambda_{D N} \geq 0$, thus confirming the Newman conjecture. Instead of looking at individual pairs of zeros, they focused on zeros in intervals of the type $[T, T+\alpha]$, where $1 \ll \alpha \ll \log T$ and proved that they exhibit a kind of relaxation to local equilibrium - if $\Lambda_{D N}<0$, then the zeros of $H_{0}$ would be arranged locally as an approximate arithmetic progression. To illustrate, observe that the function

$$
H_{\lambda}(z)=\int_{-\infty}^{\infty} e^{\lambda t^{2}} \Phi(t) e^{i z t} d t
$$

satisfies the backward heat equation,

$$
\partial_{t} H_{t}=-\partial_{z z}^{2} H_{t},
$$

with terminal condition $H_{0}$. This was noted, perhaps for the first time, in CSV94, where it was also observed that for $t>\Lambda_{D N}$, the real zeros $x_{k}(t)$ obey the system of ODEs,

$$
\partial_{t} x_{k}=2 \sum_{j \neq k} \frac{1}{x_{k}-x_{j}} .
$$

A one-parameter family of solutions to (22), called the equilibrium state, is given by $H_{t}(z)=e^{t u^{2}} \cos z u$, for $u>0$, whose zeros are all arranged as an arithmetic progression $\left\{\frac{2 \pi\left(k+\frac{1}{2}\right)}{u}: k \in \mathbb{Z}\right\}$. As discussed in [RoT18, Section 4], (23) is reminiscent of Dyson Brownian motion and of similar behavior that was studied by Erdös, Schlein, and Yau ESY11, in the context of gradient flow for the eigenvalues of random matrices.

We now sketch some of the main steps in RoT18. Assuming that $\Lambda_{D N}<0$ (and hence also assuming the validity of the $\mathrm{RH}$ ), their goal was to show a contradiction by obtaining some control on the distribution of the zeros of $H_{t}$ when $\Lambda_{D N} / 2 \leq$ 
$t \leq 0$. To do this, they exploit an observation in CSV94 that the dynamics for $H_{t}$ induces a gradient flow for the zeros $\left\{x_{j}(t)\right\}$. Indeed, for $\Lambda_{D N}<t \leq 0$, one can define a Hamiltonian by

$$
\mathcal{H}(t)=\sum_{j, k: j \neq k} \log \frac{1}{\left|x_{j}(t)-x_{k}(t)\right|}
$$

and then view the evolution of $H_{t}$ as the gradient flow of $\mathcal{H}$,

$$
\partial_{t} \mathcal{H}(t)=-4 E(t),
$$

where $E(t)$ is defined, roughly speaking, as

$$
E(t)=\sum_{j \neq k} \frac{1}{\left|x_{j}(t)-x_{k}(t)\right|^{2}} .
$$

Since (25) is only a formal sum, Rodgers and Tao studied a mollified version and used that together with (24) to show that $\left\{x_{j}(t)\right\}$ at $t=0$ would satisfy

$$
x_{j+1}(0)-x_{j}(0)=\frac{4 \pi+o_{T}(1)}{\log T}, \quad \text { if } j \in[T \log T, 2 T \log T],
$$

for a fraction $1-o(1)$ of these zeros - see [RoT18, Sec. 9]. This would imply (under the assumption $\Lambda_{D N}<0$ ) that the spacings between the zeros of the zeta function are rarely much larger or much smaller than in an arithmetic progression. However this would contradict a consequence of a result of Montgomery [Mon73], who analyzed the pair correlations for the zeros assuming the RH. The results of Mon73] imply that a positive proportion of the spacings are strictly smaller than the mean spacing; see also CGG85. That completes our very rough outline of their proof.

2.7. The upper bound for $\Lambda_{D N}$. Recall that the RH is equivalent to the inequality $\Lambda_{D N} \leq 0$ : Based on the properties of universal multipliers, de Bruijn proved the upper bound $\Lambda_{D N} \leq 1 / 2$. This has been improved more recently by Ki, Kim, and Lee [KKL09] to $\Lambda_{D N}<1 / 2$ along with other results, as we now discuss.

The starting observation of KKL09 is that $S(t)=e^{\lambda t^{2}}$ is indeed a strong universal multiplier in de Bruijn's sense, namely, if for some $\varepsilon>0$, all but a finite number of roots of (9) lie in the strip $|\Im z| \leq \varepsilon$, then for any $\lambda>0$, the function (16) has only a finite number of nonreal roots. A further observation by KKL09] is that for certain functions $F$ (roughly speaking, those where the number of nonreal zeros of (9) is less than the number of real zeros), the equality in (17) cannot be reached. More precisely:

Theorem 13. Suppose that $f(z)=\int_{-\infty}^{\infty} F(t) e^{i z t} d t$ is a real entire function of order less than $2, f$ has finitely many nonreal zeros, and the number of nonreal zeros of $f$ in the upper half-plane does not exceed the number of real zeros. Suppose also that $\Delta_{0}>0$ and the zeros of $f$ lie in the strip $\left\{z:|\Im z| \leq \Delta_{0}\right\}$. If $\lambda \in\left(0, \Delta_{0}^{2} / 2\right)$, then the zeros of

$$
\int_{-\infty}^{\infty} F(t) e^{\lambda t^{2}} e^{i z t} d t
$$

lie in $\left\{z:|\Im z| \leq \Delta_{1}\right\}$ for some $\Delta_{1}<\sqrt{\Delta_{0}^{2}-2 \lambda}$.

Then, using saddle-point methods and the properties of strong universal multipliers, KKL09] proved the following. 
Theorem 14. For any $\lambda>0$, all but finitely many zeros of $H_{\lambda}(\cdot)$ are real and simple.

Note that $H_{\lambda}$ has infinitely many real zeros by results of Pólya [P26] (see Proposition 4 above and the preceding discussion). Combining this with Theorem 13, one concludes the upper bound $\Lambda_{D N}<1 / 2$. The proof presented in KKL09 suggested but did not give a quantitative improvement for $\Lambda_{D N}$ beyond $\Lambda_{D N}<1 / 2$.

Improved upper bounds for $\Lambda_{D N}$ have been discussed in detail by Tao and collaborators in the Polymath 15 project [T18]. They combine the methods of [DB50] and [KKL09] with extensive numerical computations; at the time of the preparation of this survey paper, the upper bound was $\Lambda_{D N} \leq 0.22$.

\section{De Bruijn-Newman constant for general measures}

In this section we study the function $H_{\mu, \lambda}(z)$ defined as in (11) for a general even probability measure $\mu$. We will classify $\mu$ 's according to the zeros of $H_{\mu, \lambda}(z)$ and the de Bruijn-Newman constant $\Lambda_{D N}(\mu)$, defined as follows. As in section 1, we define

$$
\mathcal{P}_{\mu}:=\left\{\lambda: H_{\mu, \lambda}(z) \text { is entire and has only real zeros }\right\} .
$$

If $\mathcal{P}_{\mu}$ is nonempty, then $\Lambda_{D N}(\mu)$ is defined as its infimum. If $\mathcal{P}_{\mu}$ is empty but $H_{\mu, \lambda}(z)$ is entire for all $\lambda$, we define $\Lambda_{D N}(\mu)$ to be $+\infty$; in the remaining case, $\Lambda_{D N}(\mu)$ is undefined. When $d \mu(t)=f(t) d t$, we write $\Lambda_{D N}(f)$ and $\mathcal{P}_{f}$. Two main ingredients we use are Theorem [16 (proved in [NeW17]), based on the only real zeros property being preserved under convergence, and the properties of strong universal factors (e.g., Theorem 7) studied by de Bruijn and others.

3.1. A weak convergence theorem. Let $\mu$ be a probability measure on $\mathbb{R}$, and let $X$ be a random variable on some probability space $(\Omega, \mathcal{F}, \mathbb{P})$ with distribution $\mu$. Motivated by the statistical physics results discussed in subsection 2.5. we define a collection $\mathcal{X}$ of probability measures as follows. Here we use the standard probability theory notation with $\mathbb{E}[h(X)]$ denoting $\int_{-\infty}^{+\infty} h(t) d \mu(t)$.

Definition 15. We say $\mu$ ( or $X$ ) is in $\mathcal{X}$ if it has the following three properties:

(1) $X$ has the same distribution as $-X$,

(2) $\mathbb{E}\left[\exp \left(b X^{2}\right)\right]<\infty$ for some $b>0$,

(3) the function $\mathbb{E}[\exp (i z X)]$, defined for all $z \in \mathbb{C}$, has only real zeros.

The next theorem, from [NeW17], states that the combination of these three properties (and in particular, the only real zeros property of the Fourier transform) is preserved under weak convergence (i.e., pointwise convergence of the Fourier transform on the real axis). It helps explain why the sub-Gaussian property (2) is built into Definition 15 .

Theorem 16. Suppose for each $n \in \mathbb{N}, \mu_{n} \in \mathcal{X}$ and $\mu_{n}$ converges weakly to the probability measure $\mu$. Then $\mu \in \mathcal{X}$.

This theorem seems surprising at first glance for the following reason. Since $\mu_{n} \in \mathcal{X}$ for each $n$, there is some $b_{n}>0$ so that $X_{n}$ distributed by $\mu_{n}$ satisfies $\mathbb{E}\left[\exp \left(b_{n} X^{2}\right)\right]<\infty$. But without assuming that $b_{n}$ is bounded away from zero as $n \rightarrow \infty$, why should it be that the limit $X$ has $\mathbb{E}\left[\exp \left(b X^{2}\right)\right]<\infty$ for some $b>0$ ? The answer, in brief, is that the purely real zeros property (3) of Definition 15 
somehow implies that $b_{n}$ can be bounded away from zero or else weak convergence would fail.

The key to the proof of Theorem 16 is the product representation, for $X \in \mathcal{X}$,

$$
\mathbb{E}[\exp (i z X)]=e^{-B z^{2}} \prod_{k}\left(1-z^{2} / y_{k}^{2}\right),
$$

with $B \geq 0, y_{k} \in(0, \infty)$ and

$$
\mathbb{E}\left[X^{2}\right]=2\left(B+\sum_{k}\left(1 / y_{k}^{2}\right)\right)<\infty .
$$

One then shows that weak convergence for $X_{n} \in \mathcal{X}$ (distributed by $\mu_{n}$ ) requires a uniform bound first for $\mathbb{E}\left[X^{2}\right]$ and then that this yields a uniform bound away from zero for the sequence $\left\{b_{n}\right\}$ discussed above.

The next theorem, proved in NeW17, relates the all real zeros property to the distribution tail behavior and explains further why the sub-Gaussian property (2) is natural in Definition 15. The theorem follows directly from a theorem of Goldberg and Ostrovskii GO74.

Theorem 17. Suppose the random variable $X$ satisfies the following two properties:

(1) $\mathbb{E}\left[e^{b|X|^{a}}\right]<\infty$ for some $b>0$ and $a>1$,

(2) $\mathbb{E}\left[e^{b^{\prime} X^{2}}\right]=\infty$ for all $b^{\prime}>0$.

Then $\mathbb{E}\left[e^{i z X}\right]$ has some zeroes that are not purely real.

One can derive from Theorem 16] a different result than Theorem 17, which also shows that the Fourier transform of certain distributions do have some nonreal zeros.

Proposition 18. Let $\rho$ be an even probability measure such that $\int_{-\infty}^{\infty} e^{b t^{2}} d \rho(t)=$ $\infty$, for any $b>0$. Then for any $\lambda<0$,

$$
G_{\lambda}(z):=\int_{-\infty}^{\infty} e^{i z t} e^{\lambda t^{2}} d \rho(t)
$$

has some zeros that are not real. Thus $\mathcal{P}_{\rho}$ is either $\{0\}$ and $\Lambda_{D N}(\rho)=0$ or else $\mathcal{P}_{\rho}$ is empty.

Proof. Fix $\lambda<0$ and suppose that $G_{\lambda}(z)$ has only real zeros. Take a sequence $\left\{\lambda_{n}\right\}$ such that $\lambda_{0}=\lambda,\left\{\lambda_{n}\right\}$ is increasing, and $\lambda_{n} \rightarrow 0$ as $n \rightarrow \infty$. Applying Theorem 7 we conclude that $G_{\lambda_{n}}(z)$ has only real zeros for all $n$. The measure $e^{\lambda_{n} t^{2}} d \rho(t) / G_{\lambda_{n}}(0)$ is clearly even and satisfies property (2) of Definition 15 with $b=-\lambda_{n} / 2$, and therefore $e^{\lambda_{n} t^{2}} d \rho(t) / G_{\lambda_{n}}(0) \in \mathcal{X}$. Since $e^{\lambda_{n} t^{2}} d \rho(t) / G_{\lambda_{n}}(0)$ converges weakly to $\rho$, we can apply Theorem 16 to conclude that $\rho \in \mathcal{X}$. But this contradicts the fact that $\int_{-\infty}^{\infty} e^{b t^{2}} d \rho(t)=\infty$ for any $b>0$.

As a consequence of Proposition 18, we may construct distributions whose Fourier transforms have nonreal zeros. Examples are distributions with density $g(x) e^{-\lambda x^{2}}$, with $\lambda>0, g \geq 0$ even, and $\int e^{b x^{2}} g(x) d x=\infty$ for any $b>0$. Specific cases include

$$
K e^{-a|x|-\lambda x^{2}} \quad \text { with } a>0, \lambda>0,
$$

and

$$
K\left(1+x^{2}\right)^{-\theta} e^{-\lambda x^{2}} \quad \text { with } \theta>\frac{1}{2} \text { and } \lambda>0 .
$$


3.2. Classifying probability measures by $\Lambda_{D N}(\rho)$ and $\mathcal{P}_{\rho}$. Using Theorem 16. on weak convergence, one may classify even distributions $\rho$ according to tail behavior and $\mathcal{P}_{\rho}$. In this section we give examples of the various possibilities; they are organized in three subsections according to tail behavior - see equation (3) for the definition of $\mathcal{T}_{\rho}$.

3.2.1. $\mathcal{T}_{\rho}=(-\infty, \infty)$. We further discuss three cases, according to $\mathcal{P}_{\rho}$ - see $(26)$.

Case 1. $\mathcal{P}_{\rho}=(-\infty, \infty)$.

This class of probability distributions is completely characterized by Theorem 10. either

$$
\rho(t)=\frac{1}{2}\left(\delta\left(t-t_{0}\right)+\delta\left(t+t_{0}\right)\right), \text { for some } t_{0} \geq 0,
$$

or $\rho$ is absolutely continuous with density

$$
K t^{2 m} \exp \left(-\alpha t^{4}-\beta t^{2}\right) \prod_{j}\left[\left(1+\frac{t^{2}}{a_{j}^{2}}\right) e^{-\frac{t^{2}}{a_{j}^{2}}}\right] .
$$

We note (see the discussion after Theorem 10) that for $\alpha=0, \rho$ can belong to Case 4 below.

Case 2. There exists $\Lambda_{0} \in(-\infty, \infty)$, such that $\mathcal{P}_{\rho}=\left[\Lambda_{0}, \infty\right)$.

The simplest example in this class is a $\{ \pm 1,0\}$-valued symmetric random variable, for instance

$$
\rho(t)=\frac{1}{6}(\delta(t-1)+\delta(t+1))+\frac{2}{3} \delta(t) .
$$

One can explicitly calculate $\int e^{i z x} e^{b x^{2}} d \rho=\frac{2}{3}+\frac{1}{6} e^{b} y+\frac{1}{6} e^{b} \frac{1}{y}$, where $y=e^{i t}$. When $e^{b}<2$, it has roots where $y$ is real (and $|y| \neq 1$ ); whereas for $e^{b} \geq 2$, it has roots only where $|y|=1$ (thus $t$ is real). Therefore

$$
\left\{b: \int e^{i z x} e^{b x^{2}} d \rho \text { has only real zeros }\right\}=[\ln 2, \infty) .
$$

Examples where $d \rho(x)=K f(x) d x$ follow from the results are discussed in section 2. These include $f(x)=\exp (-a \cosh (x))$ with $a>0, f(x)=\exp \left(-x^{2 q}\right)$ with $q \in\{3,4,5, \ldots\}$, and the RH case of $f(x)=\Phi(x)$.

Case 3. $\mathcal{P}_{\rho}=\emptyset$.

We begin with a general proposition that is easily seen to follow from Theorem 16] and Pólya's result that $e^{b t^{2}}$ is a universal factor.

Proposition 19. Suppose $\rho$ is even with $\mathcal{T}_{\rho}=(-\infty, \infty)$ such that for some $0<$ $b_{n} \rightarrow \infty$ and $r_{n}>0$,

$$
\left.K_{n} e^{b_{n} t^{2}} d \rho(t)\right|_{t=r_{n} u} \rightarrow d \mu(u),
$$

with $\mu \notin \mathcal{X}$, where $K_{n}$ normalizes the left-hand side to be a probability measure; then $\mathcal{P}_{\rho}=\emptyset$.

We next sketch how to construct such a $\rho$ of the form

$$
d \rho(t)=K \sum_{k=1}^{\infty} a_{k}\left(\delta\left(t-d_{k}\right)+\delta\left(t+d_{k}\right)\right),
$$


with $d_{k} \nearrow \infty$ and $a_{k} \searrow 0$ rapidly, $r_{n}=d_{n+1}$, and

$$
d \mu=(1 / 2) \delta(u)+(1 / 4) \delta(u-1)+(1 / 4) \delta(u+1) .
$$

That $\mu \notin \mathcal{X}$ follows from the explicit calculation of Case 2 .

We start with $a_{1}=1$ and inductively construct first $a_{n+1}$ then $b_{n}$ for $n \geq 1$. A key idea is that $b_{n}$ will be a solution $b$ of

$$
e^{b d_{n+1}^{2}} a_{n+1}=\sum_{k=1}^{n} e^{b d_{k}^{2}} a_{k}
$$

so that $e^{b_{n} t^{2}} d \rho$ will give equal mass to $\left\{d_{n+1}\right\}$ and $\left(0, d_{n}\right]$. Because we will also require $d_{k+1} / d_{k} \rightarrow \infty$ (say, $d_{k}=e^{k^{2}}$ ), this will lead to the limit $\mu$ of (29). The inductive choice of $a_{n+1}$ will imply that there is a unique smallest positive solution $b=B\left(a_{n+1} ; a_{1}, \ldots, a_{n}\right)$ of (30).

We now construct $a_{n}$ inductively. Choose $a_{n+1}$ as any $a>0$ (say, the largest) satisfying the following three inequalities (with the first two only for $n \geq 2$ ):

$$
\begin{gathered}
e^{b_{n-1} d_{n+1}^{2}} a_{n+1} \leq(1 /(n+1)) e^{b_{n-1} d_{n}^{2}} a_{n}, \\
B\left(a_{n+1} ; a_{1}, \ldots, a_{n}\right) \geq b_{n-1}, \\
B\left(a_{n+1} ; a_{1}, \ldots, a_{n}\right) \geq n+1 .
\end{gathered}
$$

Then, as previously indicated, we choose $b_{n}=B\left(a_{n+1} ; a_{1}, \ldots, a_{n}\right)$. This completes our sketch except to note that the $n+1$ appearing on the right-hand sides of (31) and (33) could be replaced by any sequence $\theta_{n}$ with $\theta_{n}>1$ and $\theta_{n} \nearrow \infty$.

3.2.2. $\mathcal{T}_{\rho}=\left(-\infty, b_{0}\right)$ with $b_{0} \in(0, \infty)$.

Case 4. $\mathcal{P}_{\rho}=\left(-\infty, b_{0}\right)$.

Here one can take the Gaussian measure $d \rho(s) / d s=\left(b_{0} / \pi\right)^{1 / 2} e^{-b_{0} s^{2}}$.

Case 5. There exists $\Lambda_{0} \in\left(-\infty, b_{0}\right)$ such that $\mathcal{P}_{\rho}=\left[\Lambda_{0}, b_{0}\right)$.

Take $b_{0} \in(0, \infty)$ large, and let $d \rho(t):=\frac{1}{5}(3 \delta(t)+\delta(t-1)+\delta(t+1)) d t$. Also take $d \nu_{b_{0}}(t)=\left(b_{0} / \pi\right)^{-1 / 2} e^{-b_{0} t^{2}} d t$, and let $\rho=\mu * \nu_{b_{0}}$. Then

$$
d \rho(x)=K e^{-b_{0} x^{2}}\left[3+e^{-b_{0}} e^{2 b_{0} x}+e^{-b_{0}} e^{-2 b_{0} x}\right] d x
$$

is such that $\int e^{b x^{2}} d \rho<\infty$ if and only if $b<b_{0}$. By an explicit computation,

$$
\begin{aligned}
\int e^{i z x} e^{b x^{2}} d \rho= & K e^{-\frac{z^{2}}{2} \frac{1}{2\left(b_{0}-b\right)}} \\
& \times\left[3+e^{-\left(b_{0}-\frac{b_{0}^{2}}{b_{0}-b}\right)} e^{i \frac{b_{0}}{b_{0}-b} z}+e^{-\left(b_{0}-\frac{b_{0}^{2}}{b_{0}-b}\right)} e^{-i \frac{b_{0}}{b_{0}-b} z}\right] .
\end{aligned}
$$

Let $y=e^{i \frac{b_{0}}{b_{0}-b} z}$. It is not hard to check that the roots of the equation

$$
e^{-\left(b_{0}-\frac{b_{0}^{2}}{b_{0}-b}\right)}\left(y+y^{-1}\right)+3=0
$$


all satisfy $|y|=1$ if and only if $e^{-\left(b_{0}-\frac{b_{0}^{2}}{b_{0}-b}\right)} \geq 3 / 2$, i.e., $b \geq \Lambda_{0}$ for some $\Lambda_{0}=$ $\Lambda_{0}\left(b_{0}\right)>0$. Therefore

$$
\left\{b: \int e^{i z x} e^{b x^{2}} d \rho \text { has only real zeros }\right\}=\left[\Lambda_{0}, b_{0}\right) .
$$

Case 6. $\mathcal{P}_{\rho}=\emptyset$.

Let

$$
d \rho(x)=K e^{-x^{2}}(1+x) d x .
$$

Then $e^{b x^{2}} d \rho$ is integrable on $(-\infty, \infty)$ if and only if $b<1$ so $b_{0}=1$. By an explicit computation, for any $b<1$ and letting $\alpha=1-b$, we have

$$
\begin{aligned}
\int_{-\infty}^{\infty} e^{i z x} e^{b x^{2}} d \rho(x) & =\left(1-i \frac{d}{d z}\right) \int_{-\infty}^{\infty} e^{i z x} e^{-2(1-b) x^{2} / 2} d x \\
& =C(\alpha)\left(1+i \frac{z}{2 \alpha}\right) e^{-z^{2} / 4 \alpha} .
\end{aligned}
$$

Thus $z=2 \alpha i$ is always a nonreal zero. This finishes the verification for this particular example.

3.2.3. $\mathcal{T}_{\rho}=\left(-\infty, b_{0}\right]$ with $b_{0} \in[0, \infty)$.

Case 7. It is not possible to have $\mathcal{P}_{\rho}=\left(-\infty, b_{0}\right]$ or $\left[\Lambda_{0}, b_{0}\right]$ with $\Lambda_{0} \in\left(-\infty, b_{0}\right)$.

We rule out the possibility of such probability distributions by applying the weak convergence result, Theorem [16. Indeed, suppose $\rho$ is a probability distribution that satisfies the above conditions. Then for any $\lambda \in\left(-\infty, b_{0}\right]$ or $\in\left[\Lambda_{0}, b_{0}\right]$, the normalized measure

$$
d \mu_{\lambda}=\frac{e^{\lambda x^{2}} d \rho(x)}{\int_{-\infty}^{\infty} e^{\lambda x^{2}} d \rho(x)}
$$

satisfies all three conditions of Definition 15, Let $\lambda_{n}=b_{0}-1 / n$, so that $\mu_{\lambda_{n}} \in \mathcal{X}$. Applying Theorem [16, we conclude that as $n \rightarrow \infty, \mu_{b_{0}} \in \mathcal{X}$. But this would contradict our assumptions since property (2) of Definition 15 would then imply that $\int_{-\infty}^{\infty} e^{b x^{2}} d \rho(x)<\infty$ for some $b>b_{0}$.

Case 8. $\mathcal{P}_{\rho}=\left\{b_{0}\right\}$.

We give an example with $b_{0}=0$. Let $Y$ and $Y^{\prime}$ be i.i.d. Poisson random variables with mean $1 / 2$, so that $\mathbb{E}\left[e^{z Y}\right]=e^{\frac{1}{2}\left(e^{z}-1\right)}$. Let $W=Y-Y^{\prime}$; then $W$ is a symmetric random variable with $\mathbb{E}\left[e^{z W}\right]=\exp (\cosh z-1)$. We denote by $d \mu_{W}$ its probability distribution. Take a random variable $X$ with distribution $\frac{1+x^{2}}{2} d \mu_{W}$; then

$$
\begin{aligned}
\mathbb{E}\left[e^{z X}\right] & =\frac{1}{2} \int e^{z x}\left(1+x^{2}\right) d \mu_{W}(x)=\frac{1}{2}\left(1+\frac{d^{2}}{d z^{2}}\right) \mathbb{E}\left[e^{z W}\right] \\
& =\frac{1}{2} \cosh z \cdot(1+\cosh z) \exp (\cosh z-1),
\end{aligned}
$$

whose zeroes are $\pm i \pi / 2, \pm i 3 \pi / 2, \ldots$ and $\pm i \pi, \pm i 3 \pi, \ldots$, which are all purely imaginary. The fact that for any $b<0$, some zeros of $H_{\rho}(z)$ are not purely real follows as in Case 7.

We note that one can obtain an example of Case 8 with $b_{0}>0$ by replacing the above distribution $d \mu_{X}$ with Const. $\exp \left(-b_{0} x^{2}\right) d \mu_{X}$. 
Case 9. $\mathcal{P}_{\rho}=\emptyset$.

Examples of such probability distributions have been given in the discussion after Proposition 18.

\section{ACKNOWLEDGMENTS}

We thank Ivan Corwin for the invitation to submit this paper to the Bulletin of the $A M S$ and for comments on an earlier draft. We also thank Louis-Pierre Arguin and two anonymous reviewers for their detailed comments and suggestions which have been incorporated into the current draft.

\section{ABout the AUthors}

Charles M. Newman is Silver Professor of Mathematics and Global Network Professor at NYU's Courant Institute of Mathematical Sciences and at NYU-Shanghai. His research interests focus on probability theory and statistical physics.

Wei $\mathrm{Wu}$ is assistant professor of statistics at University of Warwick. His research focus is probability theory and mathematical statistical mechanics.

\section{REFERENCES}

[ABB19] L.-P. Arguin, D. Belius, P. Bourgade, M. Radziwiłl, K. Soundararajan. Maximum of the Riemann zeta function on a short interval of the critical line, Comm. Pure Appl. Math., 72 (2019) no. 3, 500-535.

[AOR19] L.-P. Arguin, F. Ouimet, M. Radziwiłł. Moments of the Riemann zeta function on short intervals of the critical line, arXiv:1901.04061 (2019).

[BK13] P. Bourgade and J. P. Keating, Quantum chaos, random matrix theory, and the Riemann $\zeta$-function, Chaos, Prog. Math. Phys., vol. 66, Birkhäuser/Springer, Basel, 2013, pp. 125-168, DOI 10.1007/978-3-0348-0697-8_4. MR3204184

[CGG85] J. B. Conrey, A. Ghosh, D. Goldston, S. M. Gonek, and D. R. Heath-Brown, On the distribution of gaps between zeros of the zeta-function, Quart. J. Math. Oxford Ser.

(2) 36 (1985), no. 141, 43-51, DOI 10.1093/qmath/36.1.43. MR780348

[CNV88] G. Csordas, T. S. Norfolk, and R. S. Varga, A lower bound for the de Bruijn-Newman constant $\Lambda$, Numer. Math. 52 (1988), no. 5, 483-497, DOI 10.1007/BF01400887. MR 945095

[COSV93] G. Csordas, A. M. Odlyzko, W. Smith, and R. S. Varga, A new Lehmer pair of zeros and a new lower bound for the de Bruijn-Newman constant $\Lambda$, Electron. Trans. Numer. Anal. 1 (1993), no. Dec., 104-111 (electronic only). MR1253639

[CRV91] G. Csordas, A. Ruttan, and R. S. Varga, The Laguerre inequalities with applications to a problem associated with the Riemann hypothesis, Numer. Algorithms 1 (1991), no. 3, 305-329, DOI 10.1007/BF02142328. MR1135299

[CSV94] G. Csordas, W. Smith, and R. S. Varga, Lehmer pairs of zeros, the de Bruijn-Newman constant $\Lambda$, and the Riemann hypothesis, Constr. Approx. 10 (1994), no. 1, 107-129, DOI 10.1007/BF01205170. MR1260363

[DB50] N. G. de Bruijn, The roots of trigonometric integrals, Duke Math. J. 17 (1950), 197226. MR0037351

[ESY11] L. Erdős, B. Schlein, and H.-T. Yau, Universality of random matrices and local relaxation flow, Invent. Math. 185 (2011), no. 1, 75-119, DOI 10.1007/s00222-010-0302-7. MR.2810797

[FFS92] R. Fernández, J. Fröhlich, and A. D. Sokal, Random walks, critical phenomena, and triviality in quantum field theory, Texts and Monographs in Physics, Springer-Verlag, Berlin, 1992. MR.1219313 
[FK14] Y. V. Fyodorov and J. P. Keating, Freezing transitions and extreme values: random matrix theory, and disordered landscapes, Philos. Trans. R. Soc. Lond. Ser. A Math. Phys. Eng. Sci. 372 (2014), no. 2007, 20120503, 32, DOI 10.1098/rsta.2012.0503. MR 3151088

[GO74] A. A. Gol'dberg and I. V. Ostrovs'kiı̆, The growth of entire ridge functions with real zeros (Russian, with English summary), Mathematical physics and functional analysis, No. V (Russian), Akad. Nauk Ukrain. SSR Fiz.-Tehn. Inst. Nizkih Temperatur, Kharkov, 1974, pp. 3-10, 156. MR.0409809

[KKL09] H. Ki, Y.-O. Kim, and J. Lee, On the de Bruijn-Newman constant, Adv. Math. 222 (2009), no. 1, 281-306, DOI 10.1016/j.aim.2009.04.003. MR2531375

[Leh56] D. H. Lehmer, On the roots of the Riemann zeta-function, Acta Math. 95 (1956), 291-298, DOI 10.1007/BF02401102. MR0086082

[LS81] E. H. Lieb and A. D. Sokal, A general Lee-Yang theorem for one-component and multicomponent ferromagnets, Comm. Math. Phys. 80 (1981), no. 2, 153-179. MR623156

[LY52] T. D. Lee and C. N. Yang, Statistical theory of equations of state and phase transitions. II. Lattice gas and Ising model, Physical Rev. (2) 87 (1952), 410-419. MR0053029

[Mon73] H. L. Montgomery, The pair correlation of zeros of the zeta function, Analytic number theory (Proc. Sympos. Pure Math., Vol. XXIV, St. Louis Univ., St. Louis, Mo., 1972), Amer. Math. Soc., Providence, R.I., 1973, pp. 181-193. MR0337821

[Naj18] J. Najnudel, On the extreme values of the Riemann zeta function on random intervals of the critical line, Probab. Theory Related Fields 172 (2018), no. 1-2, 387-452, DOI 10.1007/s00440-017-0812-y. MR3851835

[Ne74] C. M. Newman, Zeros of the partition function for generalized Ising systems, Comm. Pure Appl. Math. 27 (1974), 143-159, DOI 10.1002/cpa.3160270203. MR0484184

[Ne76] C. M. Newman, Fourier transforms with only real zeros, Proc. Amer. Math. Soc. 61 (1976), no. 2, 245-251 (1977), DOI 10.2307/2041319. MR0434982

[Ne91] C. M. Newman, The GHS inequality and the Riemann hypothesis, Constr. Approx. 7 (1991), no. 3, 389-399, DOI 10.1007/BF01888165. MR1120411

[NeW17] C. M. Newman and W. Wu, Lee-Yang property and Gaussian multiplicative chaos, Communications in Mathematical Physics, to appear.

[NRV92] T. S. Norfolk, A. Ruttan, and R. S. Varga, A lower bound for the de Bruijn-Newman constant $\Lambda$. II, Progress in approximation theory (Tampa, FL, 1990), Springer Ser. Comput. Math., vol. 19, Springer, New York, 1992, pp. 403-418, DOI 10.1007/978-14612-2966-7_17. MR.1240792

[O00] A. M. Odlyzko, An improved bound for the de Bruijn-Newman constant, Numer. Algorithms 25 (2000), no. 1-4, 293-303, DOI 10.1023/A:1016677511798. Mathematical journey through analysis, matrix theory and scientific computation (Kent, OH, 1999). MR.1827160

[P26] G. Pólya, On the zeros of certain trigonometric integrals, J. London Math. Soc. 1 (1926), 98-99.

[P27] G. Pólya, Über trigonometrische Integrale mit nur reellen Nullstellen (German), J. Reine Angew. Math. 158 (1927), 6-18, DOI 10.1515/crll.1927.158.6. MR 1581126

[PSc14] J. Schur and G. Pólya, Über zwei Arten von Faktorenfolgen in der Theorie der algebraischen Gleichungen (German), J. Reine Angew. Math. 144 (1914), 89-113, DOI 10.1515/crll.1914.144.89. MR:1580897

[R91] H. J. J. te Riele, A new lower bound for the de Bruijn-Newman constant, Numer. Math. 58 (1991), no. 6, 661-667, DOI 10.1007/BF01385647. MR 1083527

[Rie59] B. Riemann, Ueber die anzahl der primzahlen unter einer gegebenen grosse, Ges. Math. Werke und Wissenschaftlicher Nachlaß, 2 (1859), 145-155.

[RoT18] B. Rodgers and T. Tao, The de Bruijn-Newman constant is non-negative, arXiv:1801.05914 (2018).

[SGD11] Y. Saouter, X. Gourdon, and P. Demichel, An improved lower bound for the de BruijnNewman constant, Math. Comp. 80 (2011), no. 276, 2281-2287, DOI 10.1090/S00255718-2011-02472-5. MR2813360

[SiG73] B. Simon and R. B. Griffiths, The $\left(\phi^{4}\right)_{2}$ field theory as a classical Ising model, Comm. Math. Phys. 33 (1973), 145-164. MR0428998 
[T18] T. Tao et al., Upper bounding the de Bruijn-Newman constant, available at http:// michaelnielsen.org/polymath1/index.php?title=De_Bruijn-Newman_constant (2018)

[YL52] C. N. Yang and T. D. Lee, Statistical theory of equations of state and phase transitions. I. Theory of condensation, Physical Rev. (2) 87 (1952), 404-409. MR0053028

Courant Institute, New York University; and NYU Shanghai, People's Republic of ChInA

UNIVERSity OF WARWICK, WARWICK, UNITED Kingdom 